\title{
Sensing of DNA damage, instantly activation of repairing proteins and radio sensitizers-a biophysical model
}

\begin{abstract}
The physical nature of mechanisms by which cells sense radiation-induced DNAstrand breaks remains to be elucidated. The fast induction of, for example, ATM kinase activity immediately after exposure to ionizing radiation, suggests that it acts at an early stage of signal transduction. Existing data indicate that ATM activation is not dependent on direct binding to strand breaks. However, some fundamental questions are still not responded to, such as: how ATM directly senses structure disruption in "relaxed" chromatin, and which factors determine the impressive speed and extent of the ATM response. Here, a biophysical model for the signaling mechanism of both the instant activation of repair proteins, and the recognition of a few DNA breaks by these proteins within the entire genome is proposed. The model allows for the calculation of an electromagnetic transient, produced by an electron coherent hopping current generated by the DNA damage, which is able to induce long-range activation (phosphorylation) of repair proteins almost instantly. Existing experimental evidence verifying this approach is discussed. The possible role of this study in stimulating and orientating novel applications in medical physics, as e.g. radiotherapy, is, furthermore, addressed.
\end{abstract}

Keywords: DNA radiation damage, DNA damage sensing, DNA repair, quantumcoherence hypothesis, radiation sensitizer, radiotherapy, phosphorylation, radiotherapy, ATM kinase, apoptosis, biochemical signal, static electric field, mutagenesis, chemotherapies, mammalian cells
Volume 2 Issue 5 - 2015

\author{
Arruda-Neto JDT \\ Physics Institute, University of Sao Paulo and Sao Paulo \\ Engineering College - FESP, Brazil
}

Correspondence: Arruda-Neto JDT, Physics Institute, University of Sao Paulo, Sao Paulo, SP, Brazil, and Sao Paulo Engineering College - FESP, Sao Paulo, SP, Brazil, Email arruda@if.usp.br

Received: September 29, 2015 | Published: November 17, 2015

\section{Introduction}

DNA repair machinery - the role played by the ATM kinase

Ionizing radiation is by far the most deleterious agent to our genetic material, since it is able to induce double-strand breaks (DSB) in DNA. In this sense, cells have evolved complex signaling pathways to arrest the progression of the cell cycle in the presence of DNA damage, thereby providing increased time for repair mechanisms to operate. Finally, when the burden of genomic insult is too large to be effectively met by the various cell responses, cells are able to initiate programmed cell death (apoptosis). ${ }^{1}$ It is known that the protein kinase ATM is the central player in mammalian cell response to ionizing radiation, playing an important role in the activation of cell cycle checkpoints that lead to DNA damage-induce arrest at G1/S, S, and G2/M. Following ATM activation, phosphate groups are added (phosphorylation) to several target proteins, p53 in particular. Several reviews have dealt with many properties of ATM - its autophosphorylation is an important upstream event in the activation pathway. However, it is still a debated issue whether ATM, located outside the cell nucleus, is the primary sensor of DNA damage..$^{2-10}$ Over a decade ago, in this regard, ${ }^{4}$ showed that in the normal, undamaged cell the ATM proteins are tightly linked in pairs, the dormant ATM dimmers. Following DSB formation, autophosphorylation of ATM is induced, thus separating the two ATM proteins. The authors concluded that activation of the ATM kinase appears to be an initiating event in cellular responses to irradiation toward the subsequent repair process. Additionally, these authors concluded that DNA breaks must somehow signal to ATM molecules at a distance in the cell and that, moreover, direct binding of a protein complex to DNA breaks (resulting in ATM activation) is rather unlikely. Finally, it was proposed that the introduction of DSBs causes a rapid change in some aspects of higher-order chromatin structure and that consequently this chromatin alteration would initiate ATM activation.

\section{DSB formation versus ATM activation - unanswered questions}

Jakob and collaborators (2005) performed a sophisticate experiment at the Darmstadt/Germany accelerating facility, where mammalian cells were irradiated with heavy-ion beams, aiming at the study of protein recruitment dynamics to DNA lesions. Thus, using heavy-ion irradiation to generate localized damage in the nucleus, and with the aid of immunocytochemical microscopy detection (on GFP-tagged DNA repair proteins), it was possible to observe protein translocations to sites of ion traversals. Two key conclusions of this work are: 
1. radiation-induced changes in sub nuclear structures are not dependent on large-scale chromatin movement at short time post-irradiation; and

2. time-lapse images of the GFP-coupled DNA repair protein aprataxin revealed accumulations within a few seconds at sites of ion hits, indicating a rapid recruitment to damage sites.

Interesting too, the data analysis revealed only a small radiationindependent overall movement of chromatin. This small nondirectional migration of chromatin appeared to correspond to a constrained Brownian motion. ${ }^{11}$ On the other hand, the very fast responses after generation of DNA damage show that protein translocations, consistent with DNA damage recognition, occur almost immediately. These results are at variance with propositions that the activation of signal cascades and the processing of lesions take place following DNA damage-induced rearrangements of chromatin structures. As pointed out elsewhere, ${ }^{7}$ chromatin remodeling occurs frequently as genes are expressed, and during every cell-division cycle when DNA is replicated, yet ATM seems to ignore such alterations. Therefore, a plethora of intriguing, key, and first principles questions, raised by all of these studies, still remain unanswered. It is here suggested that they can be grouped into 3 topics: signaling/sensing, navigation/recognition and timing/extent.

\section{Signaling/Sensing}

a) ATM is locked into a state in which it cannot interact and phosphorylate its protein targets. Following DNA damage, therefore, how do the individual proteins within an ATM dimmer stop inhibiting each other even though they are still in contact? In other words, what triggers the process?

b) Even though if the possibility that the MNR complex is the primary damage sensor is considered, how does it sense? Alternatively, by means of what kind of interaction is ATM recruited and activated?

\section{Navigation/Recognition}

a) How do ATM and/or the MNR complex plan their navigation routes as to reach the DNA damage site? In other words, what in these molecular motors works as a gyrocompass? Once activated, for instance, ATM may be released from the DSB site, enabling phosphorylation of considerably more distal substrates such as the chromatin-bound histone H2AX.

b) How do proteins throughout the cell nucleus respond in a coordinated way, and how are a few DSBs within three billion base pairs recognized in a matter of seconds?

\section{Timing/Extent}

a) Which factors determine the impressive speed of the ATM response? Such fast responsiveness cannot be reconciled with the presumed existence of additional proteins regulating the ATM activation with displacements governed by passive diffusion. In fact, p53 is phosphorylated by ATM outside damaged regions, apparently without needing other proteins. Actually, experimental results indicate that recruitment to damage sites occurs almost immediately. ${ }^{11}$

b) And what is to be said about the extent of the ATM response? For instance, the small amount of only 18 DSBs in the genome induces phosphorylation in well over $50 \%$ of the ATM molecules in the cell, as inferred elsewhere. ${ }^{4}$

\section{Goals-hypothesis proposition}

It is here described how an electromagnetic field would be produced at a DNA damage site, thus providing "cause-effect" answers to all the questions formulated above. This approach comprises the following sequence of events:

1. Formation of an electrical transient at the damaged site. This "flash" would be responsible for protein activation (signaling).

2. Formation of a static electric field at the DSB site, functioning as an instantaneous "marker" (recognition). This effect would define the ATM route toward the DSB location (navigation clue).

3. These two effects, transient and static electric field, would account for the impressive speed, extent, and coordination of the ATM response and repair.

\section{Formation of an electromagnetic transient around the DSB site}

The phosphates in the DNA backbone make DNA one of the most highly charged polymers known. It has linear charge density of one fundamental charge (negative) per $0.17 \mathrm{~nm}$ of its length. ${ }^{12}$ Electron transfer and transport along DNA are likely to play an important role in radiation damage and repair. As pointed out elsewhere (Yu and Song, 2001), a DNA double helix with a random base pair sequence can be viewed as an 1D disordered system. In this system, the disorder leads to electronic localization, and electron hopping between these localized impurity states along the chain are responsible for the conductivity. Electron-transfer reactions can occur under such conditions via two principal mechanisms. ${ }^{13}$

1. single-step electron-tunneling process from the donor to the acceptor, and

2. Diffusive thermal hopping.

The first process is quantum-coherent, in the sense that the electron does not exchange any energy with the molecule during the transfer. Additionally, for short-distance motion (over a few base pairs), coherent tunneling can occur but exhibits an exponential decay as a function of the traveled distance $(b)$. In fact, an exponential decay of the conductance $(C)$ with increasing distance $(b)$ has been observed directly in DNA molecules folded into hairpin shapes (Figure 1). ${ }^{14}$ Therefore, an equally strongly varying electric current is established. Well-known in Electrodynamics, an electric current generates a magnetic field, and that if this field changes with time an electric field is also induced (Figure 1). In the particular case of an electric current in damaged DNA, as depicted above, a short duration electric pulse (a transient) is produced, as shown in Figure 2. Detailed calculations are presented in the Appendix 1.

\section{The underlying static electric field at the damaged site}

DSBs also generate static electric and magnetic fields, as revealed by experiments of perturbed angular correlations of gamma rays (PAC), an alternative and elegant experimental technique to study the molecular dynamics of DNA. Quite long ago, experimental results suggested that the static electric fields are quadrupole fields. ${ }^{15,16}$ While electric transients have short duration, static electric fields persist until completion of repair, also contributing to the signaling and orientation of the repairing proteins, as discussed below. 


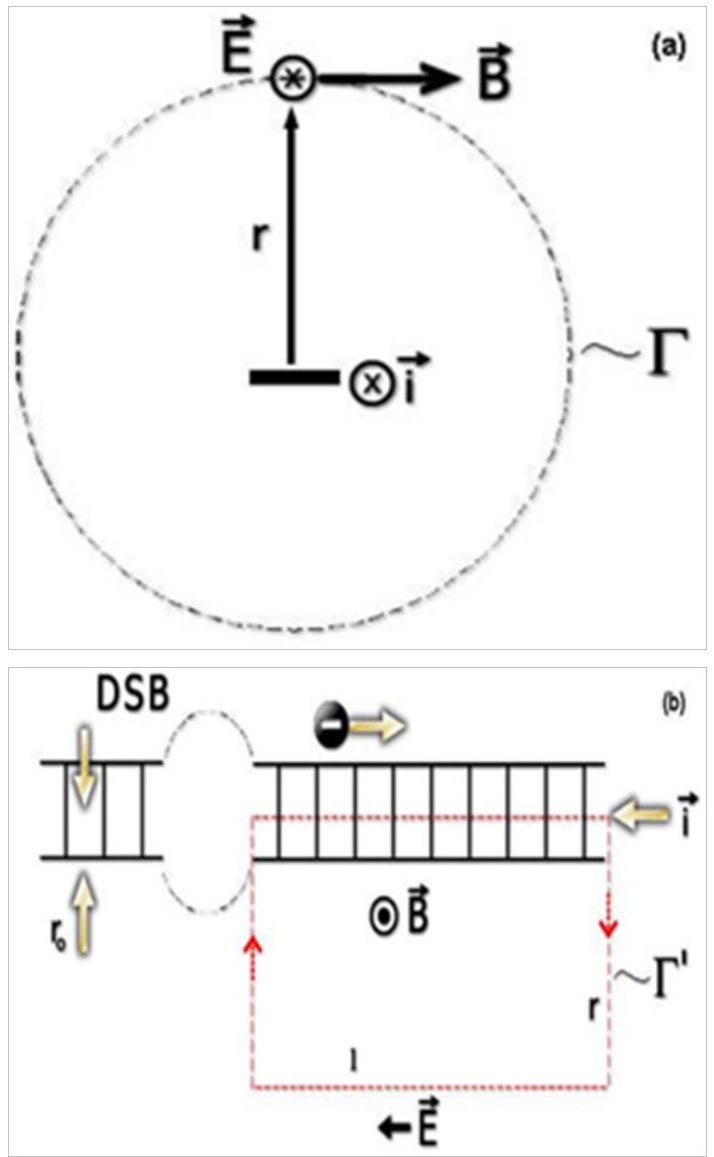

Figure I Actinin 3D structure

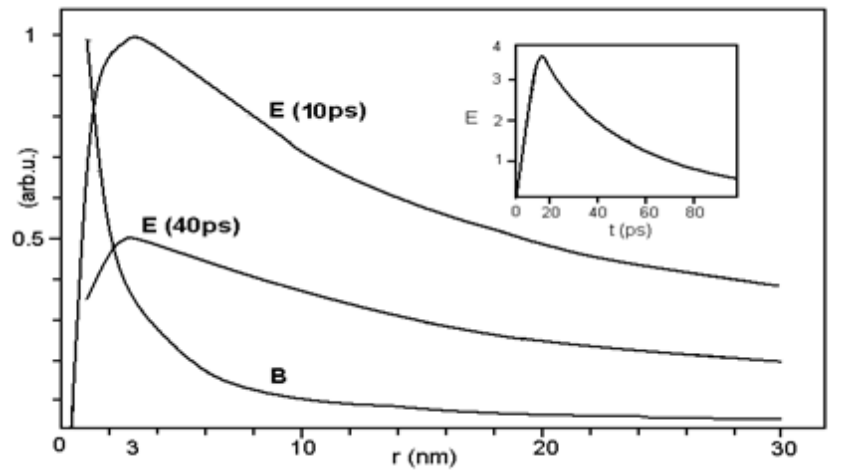

Figure 2 Results of calculations for the electric $(E)$ and magnetic $(M)$ fields as functions of the polar space coordinate $r$ see (Figure I). The results for $E$ correspond to elapsed times of 10 and 40ps after the DSB damage formation. Insert: the electric field intensity as a function of time, calculated for a fixed value of $r$.

\section{Results - answering DSB/ATM-activation questions}

\section{How are the 'dormant' dimmers "awakened"? The specificity and rapidity issues}

The ubiquity of an electric transient all over the cell volume explains much of the peculiarities observed in the repair process of radiation induced DSB. Actually, the post- DSB formation scenario, as depicted by the present approach, is the one where all points of the cell interior are experiencing an electromagnetic transient, with an intensity decreasing monotonically with the distance from the damage site, and with time duration of the order of some "biological times" (Appendix 1). It is like a flash illuminating the cellular interior. The electromagnetic nature of the signal generated by the DSB explains the rapidity of the ATM activation process, as experimentally indicated by the very fast recruitment to damage sites. ${ }^{11}$ A fundamental aspect remains to be elucidated: specificity. However, this is not a serious issue in electrodynamics, since it is certainly associated with the peculiarities of signal receiving processes, as in a radio antenna, where only those electromagnetic wave packets in resonance with the antenna receiving modes are tuned up. This insight provides a raison d'etre for the ubiquitous prevalence of helical structures in biology, which would serve as antennas for electric pulses generated at the DNA damage sites, and also generating pulses of acceleration energy on charges (mostly electrons) traversing the helices. ${ }^{17}$ Therefore, those damages generating electric pulses, capable of resonating within the helical structures of certain proteins, would trigger the repair processes conducted by these proteins, where phosphorylation is prevalent in the earlier stages. It is noted, in this regard, that the "biochemical signal" necessary to start hydrolyzation of an ATP molecule is, highly probable, an electric transient as in muscle contraction, which is triggered by an action potential at the site where a nerve impinges on the muscle-cell membrane. This quick electrical discharge releases $\mathrm{Ca}^{2+}$ ions. ${ }^{18,19}$ Under these circumstances, an electric transient activates locked ATM's following DNA damage.

\section{Navigation/Recognition}

Another unanswered key question, already mentioned above, is "how a few DSBs within three billion base pairs are recognized by the repairing enzymes (e.g. ATM and/or its substrates)".7 The most plausible answer is the inference that the gyrocompass assuring the correct navigation route is the alignment of the ATM electric dipole (which is rather huge in proteins) along with the underlying static electric field at the damage site (working as a navigation cue). Thus, the static electric field at the DSB site works as an instantaneous "marker". Such an electric sensing possibility could be at the heart of the explanation provided a decade ago by Banerjee et al., ${ }^{20}$ on how the human repair enzyme 8-oxoguanine glycosylase finds aberrant nucleotides (from oxidative damage) among the myriad of normal ones. Actually, the opening and closing of voltage-activated $\mathrm{Na}+, \mathrm{Ca} 2+$ and $\mathrm{K}+(\mathrm{Kv})$ channels underlies electrical and chemical signaling throughout biology, yet the structural basis of voltage sensing is unknown. Because of, for example, the presence of salt ions in the cytoplasm, one would argue that the net charge at each DSB site is gradually screened during the signaling-repairing time interval. However, although undergoing reorientation and attraction by the electric field toward the DSB site, ion movement is not propelled as in the repair protein; therefore, while the former moves diffusively, an ATM monomer, for example, moves processively. ${ }^{21}$

\section{Discussion-existing evidence}

Below, existing experimental data supporting the biophysical model here proposed (see section 2 - Goals) is discussed.

\section{Navigation/recognition}

As addressed above (paragraph 3.2), the underlying static electric field at the DSB site assures the correct navigation route. This is accomplished by means of the alignment of the ATM electric dipole with the static electric field at the DSB site (hypothesis at paragraph 2.2). A series of experiments was carried out at this Laboratory with prokaryotes and eukaryotes exposed to ionizing radiation and 
exogenous static electric fields. ${ }^{22-25}$ All results show that when cells exposed to radiation are immediately submitted to static electric fields, cell death increases enormously compared to the effect of radiation alone. Since the sole application of an electric (E) field has no effect on control samples, it was thus demonstrated that the E-field acts on the DNA repair process. Once the only possible effect of a static electric field is polarization, one is led to infer that repair proteins gradually tend to align their electric dipoles with the direction of the E-field. Consequently, the majority of these proteins would be unable to reach damaged sites in DNA, and lack of repair, particularly of DSB, may lead to cell death. However, a much more stringent support to this possibility was provided by measurements of $\gamma-\mathrm{H} 2 \mathrm{AX}$ foci. By the action of a static electric field on the irradiated MRC5 cells (strain of lung cells) the number of nuclei with $\gamma$-H2AX foci increased $40 \%$. These results indicate that static electric fields directly interfere in DNA repair mechanisms by inactivating cellular DNA repair processes, as revealed by the $40 \%$ surplus of nuclei with $\gamma$-H2AX foci in MRC5 cells. ${ }^{22}$

Highly revealing too, Moron et al., ${ }^{25}$ at this Laboratory, irradiated T47D breast cancer cells with 1 and $2 \mathrm{~Gy}$ of gamma radiation, and then exposed them to a static electric field of $1250 \mathrm{~V} / \mathrm{cm}$ by 24 hours. From cell cycle distributions evaluation by flow cytometry, it was found that treatment with irradiation and a static electric field (SEF) exposure causes a higher accumulation of cells at the $\mathrm{S}$ phase, and a corresponding reduction at $\mathrm{G} 1$, while the population in $\mathrm{G} 2 / \mathrm{M}$ remained nearly unchanged. It was concluded that a SEF interferes with the progression trough S-phase in irradiated cells, most likely due to an interference with DNA repair mechanisms, thus working as a radiation sensitizer for possible clinical application. It is clear that there is no possibility for a direct observation of an endogenous electric field (at a DSB site) interacting with a repairing enzyme (e.g. the ATM, in the case of mammalian cells). However, the results obtained at this Laboratory strongly indicate that, although exogenous, E-fields interfere with repairing enzymes. Therefore, the reverse process should be true, that is, through electric dipole orientation repair proteins sense the static electric field at the damage site and use it as a navigation cue. Interestingly, such a possibility complements the explanation provided by Banerjee et al., ${ }^{20}$ on how the human repair enzyme 8-oxoguanine glycosylase finds aberrant nucleotides (from oxidative damage) among the myriad of normal ones.

\section{Signaling/sensing}

Biological effects at the cellular level induced by electromagnetic radiation, as e.g. phosphorylation, are well established. Fairly interesting in this regard, over three decades ago Goodman et al., ${ }^{26}$ demonstrated that very weakly pulsing exogenous electromagnetic fields (pulse amplitude around $0.15 \mathrm{~V} / \mathrm{m}$ ) induce transcription of genes in cultured salivary gland cells of the fruitfly. In fact, weak direct currents have modified basic cellular phenomena such as growth, differentiation, dedifferentiation, and repair. Weisbrot et al., ${ }^{27}$ however, found out that low-frequency exposures on Drosophila melanogaster, during the 10-day developmental period from egg lying through pupation, induced the phosphorylation of the nuclear transcription factor ELK-1.

Therefore, the hypothesis worked out here makes strong sense, stating that the phosphorylation of head-proteins in cascade repairing reactions, as e.g. ATM in mammalian cells, MEC1 in S. cerevisiae and RAD3 in S. pombe, is induced by an electromagnetic (EM) transient emitted from the DNA damaged site. ${ }^{23}$ While providing a conceptually correct cause-effect relationship for the primary DNA signaling process, this hypothesis points to the very likely possibility that EM fields initiate phosphorylation by interacting directly with moving electrons in proteins. This is precisely the analog of electromagnetic initiation of DNA transcription. ${ }^{28}$

\section{Outlook-cancer prevention, therapy and radiation sensitizers}

As pointed out elsewhere, there is a research field on biological responsiveness to DNA damage, encompassing and integrating aspects as diverse as DNA repair, mutagenesis, programmed cell death, and damage tolerance among others. ${ }^{29}$ The development of new strategies for blocking the unwanted consequences of DNA damage, cancer in particular, is envisaged. If cancer were already installed, however, the insights presented here would facilitate development of approaches to make tumors more sensitive to radiation and chemotherapies. In fact, the main motivation of studies on the molecular basis of cancer is to develop new therapies for tumors where there is no effective treatment, or where existing therapies have significant side effects or problems. Radiation therapy, for instance, is now quite promising with the advent of hospital-based heavy ions accelerators, providing a therapy that minimizes the killing off of normal cells. However, existing therapies could be greatly enhanced if radiation doses are kept to a minimum, and their tumor cell killing efficiency is kept to a maximum. Therefore, the combination of radiation with simultaneous application of static electric fields (SEFs) in the tumor area could work in this direction. ${ }^{25}$ This biological role of SEFs as radiation sensitizers is a relevant issue, since stand-alone exposure to this exogenous physical agent is not genotoxic.

The limits of this radio sensitization process induced by SEFs were tested by carrying out viability measurements with highly radioresistant cell lines, such as Deinococcus radiodurans, one of the fiercest radioresistant organisms, exhibiting a sophisticated repair mechanism responsible for its extremophilic character. ${ }^{22,24}$ It should be noted that the present approach, using long-range electron transfer and conduction in DNA (Appendix 1) is high on the agenda of many biochemistry and biophysics research groups, as evidenced by a Special Issue section in PNAS-102-see, in particular. ${ }^{30-32}$ The hypothesis here developed, by constituting a reasonable guideline for more elaborated signaling/transduction models, is also an original and effective contribution to the research field on cellular responses to genomic insult. Therefore, the present biophysical approach points to the possible existence of another link between DNA damage, changes in chromatin structure, and downstream signaling events, which could stimulate and orientate novel studies and applications in this area. ${ }^{33-35}$

\section{Acknowledgements}

This work was partially supported by grants from CAPES and CNPq, Brazilian Foundations.

\section{Conflict of interest}

The author declares no conflict of interest.

\section{References}

1. Lodish H, Berk A, Matsudaira P, et al. Molecular Cell Biology. 4th ed. USA: Freeman \& Co; 1999.

2. Shiloh Y. ATM and related protein kinases: safeguarding genome integrity. Nat Rev Cancer. 2003;3(3):155-168.

3. Goodarzi AA, Block WD, Lees-Miller SP. The role of ATM and ATR in DNA damage-induced cell cycle control. Prog Cell Cycle Res. 2003;5:393-411. 
4. Bakkenist CJ, Kastan MB. DNA damage activates ATM through intermolecular autophosphorylation and dimer dissociation. Nature. 2003;421(6922):499-506.

5. Kozlov S, Gueven N, Keating K, et al. ATP activates ATM in vitro: importance of autophosphorylation. J Biol Chem. 2003;278(11):93099317.

6. Petrini JH, Stracker TH. The cellular response to DNA doublestrand breaks: defining the sensors and mediators. Trends Cell Biol. 2003;13(9):458-462.

7. Bartek J, Lukas J. DNA repair: Damage alerts. Nature. 2003;421(6922):486-488.

8. Garner E, Costanzo V. Studying the DNA damage response using in vitro model systems. DNA Repair (Amst). 2009;8(9):1025-1037.

9. Smith J, Tho LM, Xu N, et al. The ATM-Chk2 and ATR-Chk1 pathways in DNA damage signaling and cancer. Adv Cancer Res. 2010;108:73112 .

10. Cremona CA, Behrens A. ATM signaling and cancer. Oncogene. 2013;33(26):3351-3360.

11. Jakob B, Rudolph JH, Gueven N, et al. Live cell imaging of heavyion-induced radiation responses by beam line microscopy. Radiation Research. 2005;163(6):681-690.

12. Yu ZG, Song X. Variable range hopping and electrical conductivity along the DNA double helix. Phys Rev Lett. 2001;86(26):6018-6021.

13. Dekker C, Ratner MA. Electronic properties of DNA. Physics World. 2001;14(8):29-33.

14. Berlin YA, Burin AL, Ratner MA. Elementary steps for charge transport in DNA: thermal activation vs. tunneling. Chem Phys. 2002;275:61-74.

15. Kalfas CA, Sideris EG, Martin PW. Perturbed gamma-gamma angular correlation studies of 111 In bound to double and single stranded DNA. Int J Appl Radiat Isot. 1984;35(9):889-893.

16. Kalfas CA, Sideris EG, Loukakis GK, et al. Rotational correlation times in irradiated DNA. J of Non-Crystalline Solids. 1994;172(2):1121-1124.

17. Steele RH. Electromagnetic field generation by ATP-induced reverse electron transfer. Arch Biochem Biophys. 2003;411(1):1-18.

18. Rios E, Pizarro G. Voltage sensor of excitation-contraction coupling in skeletal muscle. Physiol Rev. 1991;71(3):849-908.

19. Ho M-W. The rainbow and the worm-The physics of organisms. 3rd ed. Singapore: World Scientific Publishing; 2008.
20. Banerjee A, Yang W, Karplus M, et al. Structure of a repair enzyme interrogating undamaged DNA elucidates recognition of damaged DNA. Nature. 2005;434(7033):612-618.

21. Okada Y, Hirokawa N. A processive single-headed motor:Kinesin superfamily protein KIF1A. Science. 1999;283(5405):1152-1157.

22. Arruda-Neto JDT, Friedberg EC, Bittencourt-Oliveira MC, et al. Static electric fields interfere in the viability of cells exposed to ionizing radiation. Int J Radiat Biol. 2009;85(4):314-321.

23. Arruda-Neto JDT, Friedberg EC, Bittencourt-Oliveira MC, et al. The role played by endogenous and exogenous electric fields in DNA signaling and repair. DNA Repair (Amst). 2010;9(4):356-357.

24. Arruda-Neto JDT, Segreto HR, Gomez JG, et al. Radio Sensitization By Static Electric Fields Is Observed In The Extremophilic Deinococcus Radiodurans Exposed To Gamma Radiation. WebmedCentral plus. 2014.

25. Moron M, Arruda J, Segreto H, et al. Cancer Cells Jointly Exposed To Gamma-radiation And Electric Field Develop S-phase Arrest. WebmedCentral. 2011:2.

26. Goodman R, Bassett CAL, Henderson AS. Pulsing electromagnetic fields induce cellular transcription. Science. 1983;220(4603):1283-1285.

27. Weisbrot D, Lin H, Ye L, et al. Effects of mobile phone radiation on reproduction and development in Drosophila melanogaster. $J$ Cell Biochem. 2003;89(1):48-55.

28. Blank M, Goodman R. Electromagnetic initiation of transcription at specific DNA sites. J Cell Biochem. 2001;81(4):689-692.

29. Friedberg EC. DNA damage and repair. Nature. 2003;421:436-440.

30. Gray HB, Winkler JR. Long-range electron transfer. PNAS 2005;102:3534-3539.

31. Hoffman BM, Celis LM, Cull DA, et al. Differential influence of dynamic processes on forward and reverse electron transfer across a protein-protein interface. PNAS. 2005;102(10):3564-3569.

32. Yavin E, Boal AK, Stemp ED, et al. Protein-DNA charge transport: Redox activation of a DNA repair protein by guanine radical. Proc Natl Acad Sci USA. 2005;102(10):3546-3551.

33. Vorobyev E. Handbook Series on Semiconductor Parameters. In: Levinshtein M, et al. editors. London: World Scientific; 1996:1.

34. Hall DB, Holmlin RE, Barton JK. Oxidative DNA damage through longrange electron transfer. Nature. 1996;382(6593):731-735.

35. Endres RG, Cox DL, Singh RRP. The quest for high-conductance DNA. Rev Mod Phys. 2004;76:195-213. 